\title{
Impact of folic acid supplementation during pregnancy on cognitive performance of children at age 11 years: preliminary results from the FASSTT Offspring study
}

\author{
A. Caffrey ${ }^{1}$, H. McNulty ${ }^{1}$, R. Irwin ${ }^{2}$, T. Cassidy $^{3}$, M. McLaughlin ${ }^{3}$, D. Lees-Murdock ${ }^{2}$, \\ C.P. Walsh ${ }^{2}$ and K. Pentieva ${ }^{1}$ \\ ${ }^{1}$ Nutrition Innovation Centre for Food and Health (NICHE), ${ }^{2}$ Genomic Medicine Research Group and ${ }^{3}$ Psychology \\ Research Institute, Ulster University, Coleraine, BT52 1SA
}

Periconceptional folic acid supplementation is widely recognised for its role in the prevention of neural tube defects. More recent evidence suggests that maternal folate throughout pregnancy may have other roles in offspring health, including neurodevelopment and cognitive performance in the first decade of life, but the evidence at this time is predominantly observational. The aim of the current study was to investigate the effect of folic acid supplementation during pregnancy on cognitive performance of the child at 11 years.

From our original Folic Acid Supplementation in the Second and Third Trimesters (FASSTT) trial in pregnancy ${ }^{(1)}$, mother-child pairs have been followed up over the past decade for the FASSTT Offspring study. The current analysis forms part of the EpiFASSTT project involving re-sampling of children, now aged 11 years. Cognitive performance was assessed using the Wechsler Intelligence Scale for Children, Fourth UK Edition (WISC-IV) ${ }^{(3)}$. The WISC-IV measures Full Scale IQ and specific domains of cognitive performance: Verbal Comprehension (VC), Perceptual Reasoning (PR), Working Memory (WM) and Processing Speed (PS). Each of these domains is comprised of further subtests (15 in total). For this analysis, test scores of children (for each WISC-IV domain and subtests) were examined in relation to folic acid supplementation of the mother during pregnancy.

The results showed no significant effect of maternal folic acid on mean (95\% CI) Full Scale IQ in children (boys or girls) at age 11 years: placebo $104.0(100 \cdot 7,107 \cdot 3)$ vs folic acid 103.1 (99.9, 106.3); ( $\mathrm{P}=0.699)$; data not shown. The significant effect of folic acid on Verbal Comprehension was evident only in female offspring (Table). Furthermore, children (both sexes) of folic acid treated mothers compared to the placebo group had significantly higher mean scores in the Cancellation subtest within Processing Speed: 93.2 (87.0, $99.5)$ vs $84.7(77.9,91.5) ;(P=0.035)$, but not in any other domains or subtests examined (data not shown).

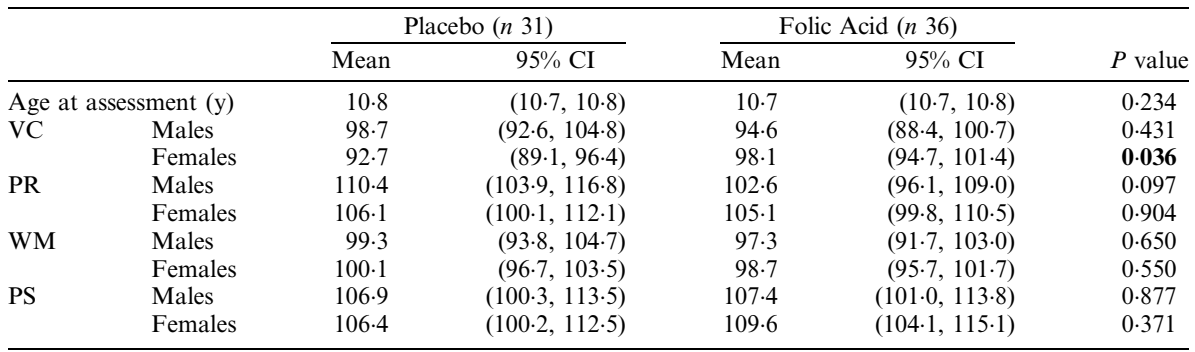

Differences between treatment groups were analysed using ANCOVA with adjustment for maternal age.

The current results are in good agreement with our earlier observations from the FASSTT Offspring study showing improved cognitive performance in these children at 3 and 7 years ${ }^{(3)}$. In addition, the findings of sex-specific effects of folic acid on cognition are also generally consistent with recent epigenetic results in cord blood of these children, which showed sex-specific changes in DNA methylation of genes related to brain development and function ${ }^{(4)}$.

These results indicate that folic acid supplementation throughout pregnancy may improve offspring performance of specific domains related to cognition. The current results however are preliminary and no firm conclusions can be made until dietary and nutrient biomarker analysis in children is completed.

The EpiFASSTT project is joint funded by the BBSRC and ESRC. For more details visit: www.bristol.ac.uk/essn.

1. McNulty B, Pentieva K, Marshall B et al. (2013) Am J Clin Nutr 98, 92-8.

2. Wechsler D (2004) Wechsler Intelligence Scale for Children Fourth Edition. Pearson: London.

3. McGarel C, McNulty H, Strain JJ et al. (2014) Proc Nutr Soc 73, E49.

4. Caffrey A, Irwin R, McNulty $\mathrm{H}$ et al. (2018) Am J Clin Nutr (In Press). 